\title{
Sarcomas de partes moles nos membros, mais comuns e tão graves quanto os sarcomas ósseos*
}

\section{Extremities Soft Tissue Sarcomas, more Common and as Dangerous as Bone Sarcomas}

\author{
Karen Voltan ${ }^{10} \quad$ André Mathias Baptista $^{2}{ }^{(0)}$ Maurício Etchebehere ${ }^{1 \odot}$ \\ ${ }^{1}$ Departamento de Ortopedia e Traumatologia, Faculdade de Ciências \\ Médicas da Universidade Estadual de Campinas, Campinas, SP, Brasil \\ 2 Instituto de Ortopedia e Traumatologia, Hospital das Clinicas, Faculdade \\ de Medicina, Universidade de São Paulo, São Paulo, SP, Brasil \\ Rev Bras Ortop 2021;56(4):419-424. \\ Endereço para correspondência Karen Voltan, MD, Departamento de \\ Ortopedia e Traumatologia, Faculdade de Ciências Médicas da \\ Universidade Estadual de Campinas (FCM-UNICAMP), Rua Tessália \\ Vieira de Camargo, 126, Cidade Universitária, Campinas, \\ SP, 13083-887, Brasil (e-mail: karenvoltan@hotmail.com; \\ karenvoltan@gmail.com; kvoltan@icloud.com).
}

\section{Resumo \\ Palavras-chave \\ - epidemiologia \\ - detecção precoce de cancer \\ - metástase \\ - neoplasia \\ - tecido ósseo \\ - fatores prognósticos \\ - sarcoma de tecidos moles}

Abstract
Os sarcomas musculoesqueléticos são doenças raras que exigem atenção. Frequentemente, apresentam alto grau de malignidade ao diagnóstico e se subestimados podem evoluir de forma agressiva local e sistemicamente. Apresentam-se como sarcoma de partes moles e sarcomas ósseos, sendo os de partes moles quatro a cinco vezes mais comuns. A maioria dos sarcomas de partes moles ocorre nos membros. Os subtipos mais comuns nas crianças e adolescentes são o rabdomiossarcoma e o sarcoma sinovial, nos adultos o sarcoma pleomórfico indiferenciado, lipossarcoma, leiomiossarcoma, mixofibrossarcoma e sarcoma sinovial; todos de alto grau de malignidade histológica. Muitos sarcomas de partes moles são confundidos com tumores benignos de partes moles, 100 vezes mais comuns, por isso são ressecados sem o planejamento necessário, acarretando em amputação de um membro que poderia ter sido preservado. Como em todos os cânceres, o fator prognóstico mais importante é a doença metastática. Na sua vigência, a taxa de sobrevida global cai em torno de 20 a $30 \%$. As taxas de sobrevida no geral são parecidas entre os sarcomas ósseos e de partes moles, portanto o sarcoma de partes moles, além de mais prevalente, mostra-se tão agressivo quanto os sarcomas ósseos, por isso merece muita atenção dos ortopedistas que frequentemente são a primeira linha de atendimento dos portadores destes tumores.

Musculoskeletal sarcomas are rare diseases that require attention. They often present a high degree of malignancy at diagnosis and, if underestimated, they can evolve
Trabalho desenvolvido no Departamento de Ortopedia e Traumatologia, Faculdade de Ciências Médicas da Universidade Estadual de Campinas (FCM-UNICAMP), Campinas, SP, Brasil. recebido

24 de Setembro de 2019

aceito

20 de Fevereiro de 2020

Publicado on-line

Setembro 25, 2020
DOI https://doi.org/

$10.1055 / \mathrm{s}-0040-1712136$.

ISSN 0102-3616. (c) 2020. Sociedade Brasileira de Ortopedia e Traumatologia. All rights reserved.

This is an open access article published by Thieme under the terms of the Creative Commons Attribution-NonDerivative-NonCommercial-License, permitting copying and reproduction so long as the original work is given appropriate credit. Contents may not be used for commercial purposes, or adapted, remixed, transformed or built upon. (https://creativecommons.org/ licenses/by-nc-nd/4.0/)

Thieme Revinter Publicações Ltda., Rua do Matoso 170, Rio de Janeiro, RJ, CEP 20270-135, Brazil 
Keywords

- epidemiology

- early detection of cancer

- metastasis

- neoplasm

- bone tissue

- prognostic factors

- soft tissue sarcoma aggressively locally and systemically. They present as soft tissue sarcoma and bone sarcomas, with soft tissue being four to five times more common. Most soft tissue sarcomas occur in the limbs. The most common subtypes in children and adolescents are rhabdomyosarcoma and synovial sarcoma, in adults undifferentiated pleomorphic sarcoma, liposarcoma, leiomyosarcoma, myxofibrosarcoma and synovial sarcoma; all with a high degree of histological malignancy. Many soft tissue sarcomas are confused with benign soft tissue tumors, 100 times more common, so they are resected without the necessary planning, resulting in amputation of a limb that could have been preserved. As in all cancers, the most important prognostic factor is metastatic disease. In its lifetime, the overall survival rate falls by $\sim 20$ to $30 \%$. Survival rates are generally similar between bone and soft tissue sarcomas, so soft tissue sarcoma, in addition to being more prevalent, is as aggressive as bone sarcomas, so it deserves a lot of attention from orthopedists, who are often the first line of care of carriers of these tumors.

\section{Introdução}

Os sarcomas musculoesqueléticos são doenças raras, de origem mesenquimal, presentes em qualquer idade e que se desenvolvem em qualquer lugar do corpo. Estas neoplasias são classificadas como sarcomas de partes moles (SPM) ou sarcomas ósseos.

Em 2013, a Organização Mundial de Saúde (OMS) descreveu mais de 50 tipos de sarcomas de partes moles e cerca de 30 tipos ósseos. ${ }^{1}$ Atualmente, os sarcomas são um grupo heterogêneo de mais de 100 tumores. $^{2}$

\section{Epidemiologia}

Apesar dessa grande variedade, os sarcomas musculoesqueléticos correspondem $\mathrm{a}<1 \%$ dos tumores malignos nos adultos e a cerca de $15 \%$ dos tumores malignos pediátri$\cos ^{1,3-5}$; assim, os SPM correspondem $a<1 \%$ de todos os tumores malignos. Os sarcomas ósseos são ainda mais raros e correspondem a cerca de $0,2 \%{ }^{1,6}$ Portanto, os SPM são 4 a 5 vezes mais comuns do que os sarcomas ósseos.

A taxa mundial da incidência dos SPM varia de 1.8 a 5.0 por 100.000 pessoas/ano. ${ }^{7}$ Podem ocorrer em qualquer idade, embora mais de 65\% desses sarcomas ocorram em pessoas $>65$ anos. $^{5}$ De acordo com o Scandinavian Sarcoma Group (SSG, na sigla em inglês) no período de 1987 a 2011 foram registrados 6.027 casos de sarcomas de partes moles a uma taxa de 1.5 a 2.0 por 100.000 habitantes/ano. ${ }^{8}$ No Japão, o Bone and Soft Tissue Tumor (BSTT, na sigla em inglês), órgão de registro de câncer específico em todo o país para tumor ósseo e partes moles registrou, no período de 2006 a 2012, 44.709 pacientes com tumores musculoesqueléticos benignos e malignos. Destes, 2.773 eram ósseos malignos e 8.228 eram malignos de partes moles. ${ }^{9,10}$ Em 2004, foram diagnosticados nos EUA cerca de 8.700 casos novos de SPM e 1.500 no Reino Unido. ${ }^{5}$ Na Europa, a incidência dos sarcomas em geral é de 6 casos por 100.000 , com 28.000 casos por ano. Em 2008, os SPM representaram quase $87 \%$ de todos os sarcomas. ${ }^{11}$ The American Cancer Society estima que em 2019 haverá nos EUA aproximadamente 1.762 .450 novos casos de câncer e acontecerão 606.880 óbitos pela doença. ${ }^{4}$ Desses casos, cerca de 3.500 acometerão os ossos e as articulações e 1.660 irão a óbito, já a incidência dos tumores de partes moles será de 12.750 casos com aproximadamente 5.270 óbitos. $^{4}$

Durante sua carreira, um ortopedista geral vai se deparar no mínimo com um osteossarcoma ou um sarcoma de Ewing. ${ }^{12}$ Tendo em vista a maior prevalência dos SPM, um ortopedista geral deve se deparar com quatro ou mais destas neoplasias.

\section{Aspectos Clínicos}

Os sarcomas musculoesqueléticos são um grupo heterogêneo de tumor com diferentes manifestações clínicas, localizações, evoluções e condutas. Aproximadamente 75\% dos SPM estão localizados nos membros e cinturas pélvica e escapular. A coxa é o local mais acometido. ${ }^{1}$ Cerca de $10 \%$ dos casos acometem o tronco e o retroperitônio. ${ }^{1}$

Na infância e na adolescência, os tumores malignos mais frequentes de partes moles são o rabdomiossarcoma e o sarcoma sinovial. ${ }^{13} \mathrm{Na}$ fase adulta, os SPM mais frequentes são sarcoma pleomórfico indiferenciado, lipossarcoma, leiomiossarcoma, mixofibrossarcoma e sarcoma sinovial. ${ }^{1,6} \mathrm{~A}$ doença metastática é a principal causa de morte relacionada aos sarcomas. As metástases dos sarcomas de tecidos moles e dos sarcomas ósseos de alto grau disseminam-se para os pulmões pela via hematogênica. ${ }^{14}$ Como cerca de três quartos dos SPM são histologicamente classificados como alto grau de malignidade, ${ }^{1}$ durante a investigação diagnóstica e estadiamento, espera-se que $10 \%$ dos pacientes apresentem metástases predominantemente para os pulmões. ${ }^{1}$ Durante a evolução, cerca de $50 \%$ dos pacientes portadores de SPM desenvolvem metástases à distância com média de sobrevida global de 12 a 18 meses. Dos portadores de metástases, $<20 \%$ estarão vivos em dois anos. ${ }^{15}$ Além da presença de metástases, as margens cirúrgicas desses sarcomas possuem grande impacto no prognóstico. ${ }^{16,17}$

Apesar de menos frequentes no grupo pediátrico, os SPM podem acometer esta faixa etária, e o rabdomiossarcoma é o SPM mais comum. Aproximadamente $50 \%$ dos casos de SPM no grupo pediátrico é rabdomiossarcoma, e os subtipos embrionário (cabeça, pescoço e trato geniturinário) e o alveolar (tórax 
e membros) são os mais comuns. Já o rabdomiossarcoma botrióide e outros subtipos são mais raros. ${ }^{18} \mathrm{O}$ rabdomiossarcoma embrionário tem como característica sua apresentação bimodal, ou seja, apresenta seus picos de incidência nos pacientes com idade de 2 a 6 anos e dos 15 aos 19 anos. $^{18,19}$ O subtipo alveolar é mais comum nos adolescentes. ${ }^{18}$

Os maiores problemas em relação à abordagem dos SPM ocorrem devido a conceitos equivocados que ainda fazem parte da prática clínica. Um deles é que tumores malignos de tecidos moles precisam ser dolorosos, apresentar crescimento rápido e serem profundos. Na verdade, podem crescer lentamente, serem indolores e localizados superficialmente. ${ }^{20}$ Por isso, o quadro clínico pode ser arrastado e, mesmo em países desenvolvidos, o encaminhamento para os serviços de referência pode levar de 6 a14 meses. ${ }^{21}$

\section{Diagnóstico e Estadiamento}

Nos centros oncológicos de referência, os pacientes são cuidados por equipes multidisciplinares. Em um mesmo serviço serão realizados exames para estadiamento local e à distância. Neste sentido, a ressonância magnética (RM) é mandatória. A biópsia ainda é necessária na maioria dos pacientes, entretanto a biópsia pode não ser realizada em tumores de partes moles que na RM apresentem característica obviamente adiposa.

A $8^{\circ}$ edição do Cancer Manual Staging da American Joint Committee on Cancer (AJCC, na sigla em inglês) atualizou em 2018 o estadiamento dos tumores de partes moles (TNM). Neste sistema é considerado o grau histológico do tumor, o tamanho, presença de linfonodos e presença de metástases. A diferenciação entre tumores superficiais e profundos deixa de existir. O T (tamanho) apresenta quatro níveis (T1 a T4) variando em $<5 \mathrm{~cm}$, até $10 \mathrm{~cm}$, até $15 \mathrm{~cm}$ e $>15 \mathrm{~cm}$. O N avalia acometimento linfonodal regional e o $\mathbf{M}$ metástases à distância. Nos sarcomas, a presença de comprometimento de linfonodos é considerada acometimento sistêmico pela neoplasia e possui a mesma importância de uma metástase à distância. Assim, N0 e M0 não há acometimento sistêmico, mas N1 ou M1 considera-se acometimento sistêmico. Os pacientes são classificados em 4 estágios, de I a IV, com algumas subdivisões. Os tumores que apresentarem N1 ou M1 são classificados no estadio IV, o mais avançado. ${ }^{22}$

O grupo de Birmingham descreveu um critério que estima a probabilidade de malignidade dos tumores de partes moles: tumor ser $>4,3 \mathrm{~cm}$ (tamanho de uma bola de golfe), aumento progressivo de tamanho, profundo à fáscia e presença de dor. Destes critérios, os dois primeiros são os de maior importância. ${ }^{23}$ Com três ou quatro critérios positivos, há uma possibilidade $>80 \%$ da lesão de partes moles ser maligna. ${ }^{24}$

Ao deparar-se com uma lesão de partes moles, a RM do segmento afetado com contraste (gadolínio) é o principal exame de imagem para sua avaliação. Através dela é possível avaliar características do tumor, sua extensão e definir o melhor local para a realização da biópsia. As principais características a serem consideradas na ressonância magnéticas são: se o tumor é sólido e se há presença de tecido adiposo, assim qualquer lesão de partes moles que não seja cística e não apresente sinal de gordura homogênea na ressonância magnética deve ser abordada potencialmente como um sarcoma (-Figura 1). O cirurgião deve planejar a via de acesso e a técnica da biópsia para permitir que todo o trajeto seja ressecado em bloco juntamente com o tumor, portanto procedimentos com vias de acesso transversais ou acompanhando linhas de força devem ser evitados quando as biópsias forem realizadas nos membros. A incisão da biópsia deve acompanhar o sentido das fibras musculares e violar o menor número possível de compartimentos (-Figura 2). Atualmente, muitas das biópsias são executadas com agulha e guiadas por ultrassonografia ou tomografia computadorizada (TC). Isso exige entrosamento do cirurgião e do médico radiologista intervencionista para garantir que o procedimento seja executado na via de acesso adequada.

O estadiamento deve ser realizado antes da ressecção do tumor solicitando, no mínimo, o exame de TC para rastreamento de metástases pulmonares. ${ }^{25}$ Diversos serviços de referência em oncologia disponibilizam a tomografia por emissão de pósitrons associada a tomografia computadorizada (PET-CT) com 18F-fluorodesoxiglicose (18F-FDG) para ser utilizado no estadiamento. $O$ exame de PET-CT avalia o metabolismo de glicose, e quanto mais agressivo o tumor, maior a avidez por glicose. Por avaliar todo organismo, além

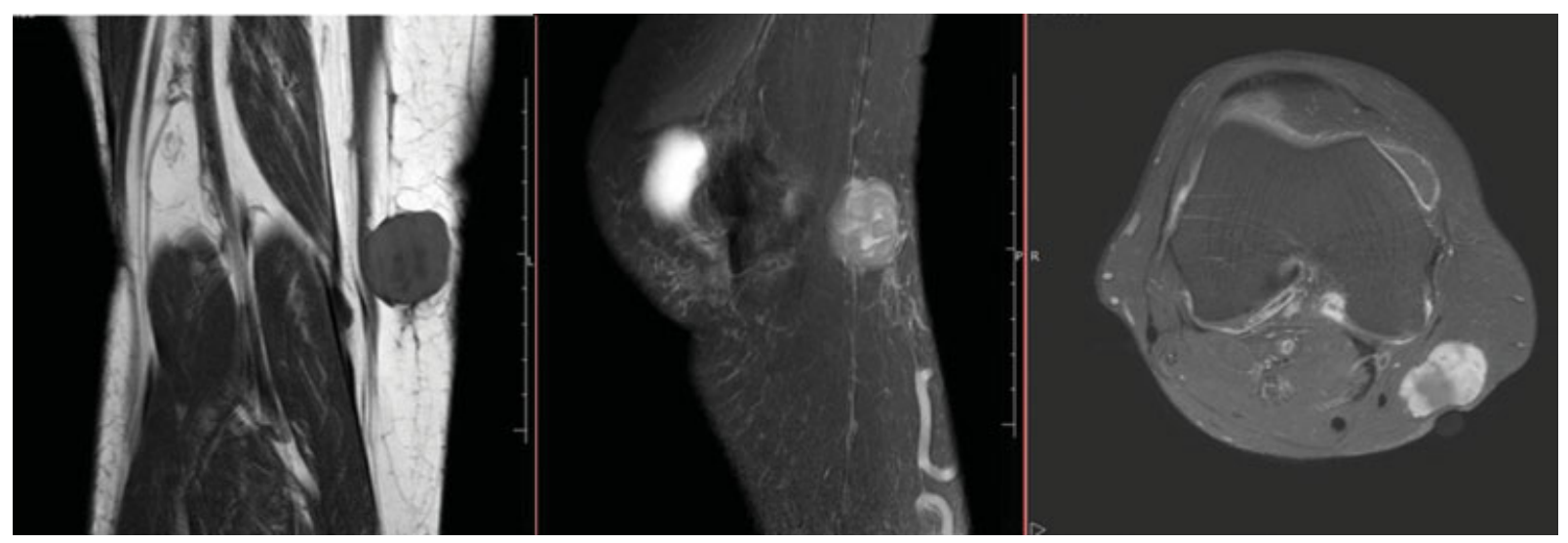

Fig. 1 Cortes de ressonância magnética de um joelho. (A) sequência coronal T1, (B) Sagital T2 SPAIR, (C) axial T1 POS GD. Observe que a lesão é sólida, relativamente pequena, tamanho próximo ao de uma bola de golfe ou de tênis de mesa. Trata-se de um SPM de alto grau. 


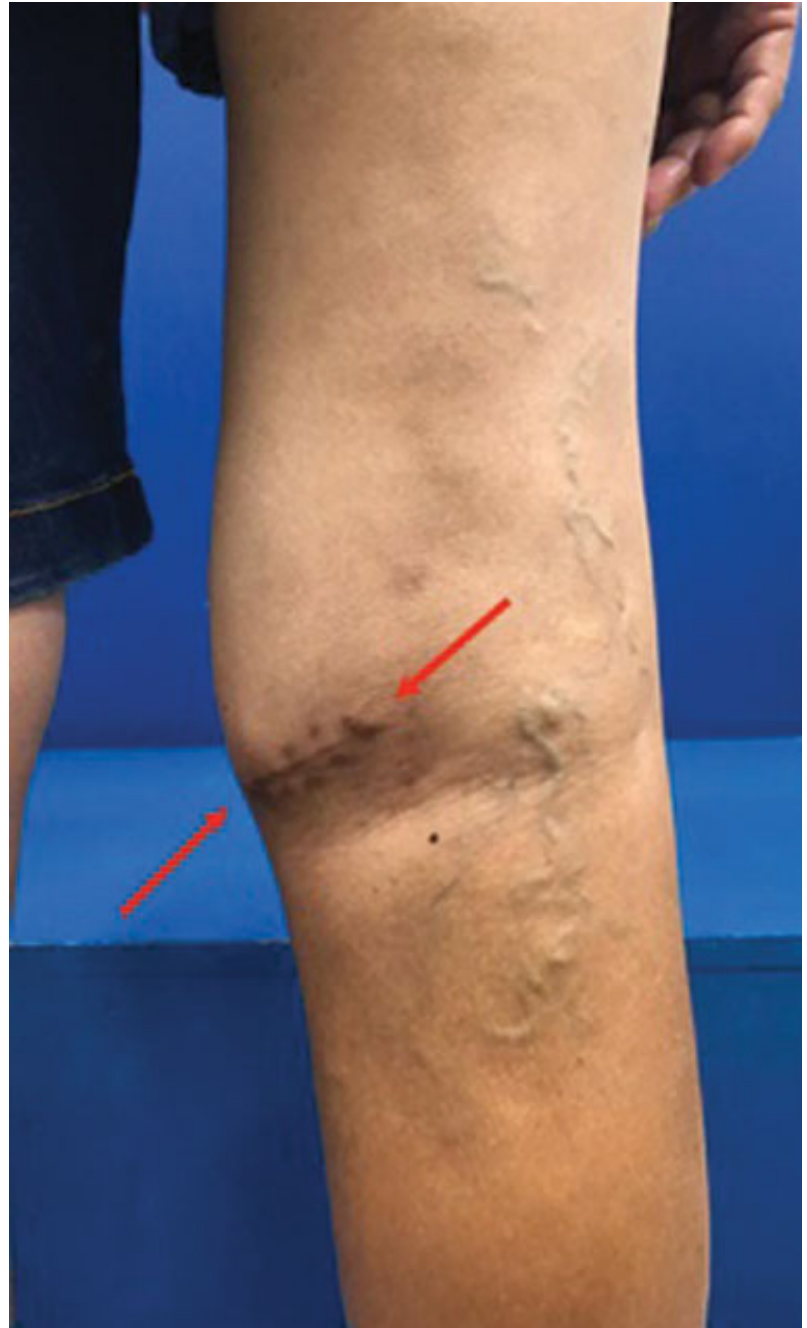

Fig. 2 Foto da mesma paciente da - Figura 1. Região posterior do joelho direito de uma paciente submetida a uma biópsia aberta com via de acesso inadequada. Observa-se cicatriz transversa que precisará ser ressecada por completo. O fechamento de pele poderá ser comprometido.

dos pulmões, o PET-CT muda o estadiamento quanto detecta metástases de diagnóstico duvidoso dentro ou fora dos pulmões. Além disso, o valor do standard uptake value (SUVmax) demonstrado no PET-CT pode predizer não só a atividade biológica, como também o grau do tumor e por isso tornou-se um fator prognóstico útil na análise de sobrevida de pacientes com SPM. O PET-CT também possui importante papel na avaliação da resposta à quimioterapia e no planejamento do volume da radioterapia. Como permite a avaliação prévia da agressividade do tumor, o PET-CT pode direcionar a realização de ressecções mais agressivas com margens adequadas e, por outro lado, pode evitar a realização de biópsias desnecessárias. ${ }^{3,11,26}$

Apesar das tentativas de evitar a realização de biópsias desnecessárias, este procedimento ainda está indicado na vasta maioria dos pacientes. 0 material para estudo anatomopatológico deve ser avaliado por um médico patologista com experiência em tumores musculoesqueléticos. Atualmente, os patologistas complementam a análise histopatológica com um painel de marcadores imunoistoquímicos.
Testes moleculares têm sido empregados para complementação do diagnóstico. Independentemente da necessidade de testes moleculares para apoiar certos diagnósticos, a análise genética detalhada do tecido tumoral de um paciente pode ter implicações importantes para a terapia direcionada. ${ }^{27}$ Técnicas como o Fluorescent In Situ Hybridization (FISH) e o Next Generation Sequencing (NGS) aos poucos estão comprovando a existência, dentro de um mesmo diagnóstico, de subtipos diferentes de tumores, com padrões muitas vezes semelhantes e que provavelmente necessitarão de terapias alvo para seu tratamento, gerando provavelmente um impacto positivo na sobrevida. ${ }^{2,27}$

\section{Tratamento}

O tratamento dos SPM depende fundamentalmente do grau histológico, da sua localização e do seu tamanho. Os protocolos utilizam quimioterapia neoadjuvante ou adjuvante e combinam o tratamento cirúrgico com a radioterapia pré- ou pósoperatória. A ressecção dos sarcomas de partes moles tem como objetivo alcançar margens cirúrgicas microscópicas livres de tumor. ${ }^{16}$ As margens de ressecção dos sarcomas são atualmente definidas como R0 margem microscópica negativa, R1 microscópica positiva, R2 margem macroscópica positiva, ou seja, permanência de tumor residual evidente. A extensão da margem R0 não é importante desde que não haja tumor residual, ${ }^{16}$ ou seja, não há diferença entre uma margem chamada exígua e uma margem de três centímetros. Apesar da busca incessante pela preservação do membro acometido por um SPM, as amputações ainda são indicadas nos casos em que as margens cirúrgicas microscópicas livres (R0) não podem ser obtidas sem que o tumor seja ressecado junto com estruturas críticas como os grandes feixes neurovasculares. Como mencionado anteriormente, pacientes que foram submetidos a ressecções não planejadas de um SPM com utilização de vias de acesso transversais apresentam maior possibilidade de serem submetidos a uma amputação. A ampliação das margens cirúrgicas nos pacientes submetidos a ressecções não planejadas de sarcomas é fundamental. Esta ampliação permite que o prognóstico de sobrevida dos pacientes submetidos aos procedimentos não planejados seja semelhante aos que se submeteram a tratamento planejado. ${ }^{28}$

Os pacientes com rabdomiossarcoma apresentam com frequência doença metastática ao diagnóstico, portanto necessitam de tratamento sistêmico neoadjuvante. A poliquimioterapia é a base do tratamento dos SPM e deve ser utilizada em todos os pacientes. Normalmente, os SPM apresentam boa resposta $(80-90 \%)$ à quimioterapia e à radioterapia, consequentemente a ressecção torna-se um procedimento mais conservador e as doses de radioterapia são reduzidas para limitar o risco de sequelas. ${ }^{13}$

\section{Prognóstico}

Os tumores de partes moles benignos, como os lipomas, são 100 vezes mais comuns do que os sarcomas de partes moles. ${ }^{23}$ Devido a sua raridade, muitos médicos e pacientes não reconhecem um SPM, o que contribui para atrasos na investigação e 
no diagnóstico. Isso ocasiona aumento do volume das lesões. $\mathrm{O}$ tamanho $<5 \mathrm{~cm}$ e a profundidade são fatores de pior prognóstico. ${ }^{29}$ Além disso, a maior incidência de tumores de partes moles benignos frequentemente leva a realização de ressecções não planejadas de sarcomas que deixam tumor residual. Hanasilo et al. relatam que os serviços de referência chegam a receber $\sim 50 \%$ dos casos novos de pacientes portadores de SPM que foram submetidos a ressecções não planejadas, e $90 \%$ desses pacientes apresentam tumor residual. ${ }^{28}$ Após uma ressecção não planejada, há maior risco de complicações e pode ser necessário uma amputação para que a margem livre de tumor seja obtida. ${ }^{23,30,31}$

A sobrevida global dos tumores musculoesqueléticos é multifatorial e depende na maioria das vezes dos fatores de risco, como subtipo histológico do tumor, grau de malignidade, idade do paciente, localização, tamanho, margens cirúrgicas alcançadas, presença de metástases, tratamento quimioterápico neoadjuvante/adjuvante e radioterapia.

Baptista et al. $^{32}$ relatam que nos pacientes com diagnóstico de sarcoma sinovial não metastático os fatores prognósticos desfavoráveis à sobrevida eram o alto grau histológico, localização (tumores proximais ao joelho ou cotovelo) e necrose espontânea de tumor $>25 \%$. A recidiva local não influenciou na sobrevida. Já Zumárraga et al. ${ }^{33}$ relataram que nos pacientes com mixofibrossarcoma um pior prognóstico da sobrevida global estava relacionado com o grau histológico do tumor, margens comprometidas, recidiva local e metástases à distância. Wu et al. ${ }^{34}$ relatam que nos lipossarcomas mixóides a idade (> 30 anos), sexo masculino, grau do tumor (alto grau) e tamanho do tumor $(>10 \mathrm{~cm}$ ) eram fatores prognósticos independentes para sobrevida global e na doença específica em pacientes com SPM das extremidades. Tsuda et al. ${ }^{35}$ relataram que em pacientes $>65$ anos as margens cirúrgicas negativas estavam associados com uma maior sobrevida. Nakamura et al. $^{36}$ relataram que a presença de inflamação sistêmica está associada a pior prognóstico. A elevação dos níveis de proteína c-reativa (PCR), hipoalbuminemia e anemia refletem o comportamento agressivo do tumor. Os dois primeiros marcadores séricos podem ser úteis para prever a sobrevida dos pacientes com doença metastática ao diagnóstico. Ishiraha et al. relataram que o grau histológico de malignidade (alto grau), o tamanho do tumor $\geq 10 \mathrm{~cm}$ e as metástases alteram o prognóstico. A recidiva local não está realmente relacionada com a ocorrência de metástases, tamanho do tumor, grau histológico e óbito. ${ }^{37}$

O rabdomiossarcoma, de modo geral, apresenta sobrevida global de $70 \%$ em 5 anos. Em várias séries pediátricas, pacientes $>10$ anos de idade foram relatados como tendo pior prognóstico do que crianças mais novas. ${ }^{13}$ Nos tumores ósseos (osteossarcoma e sarcoma de Ewing) a doença metastática diminui a sobrevida global para entre 20 e $30 \%$. Nos osteossarcomas, cerca de 15 a $20 \%$ dos pacientes já apresentam evidências macroscópicas de metástases ao diagnóstico, e de 15 a 35\% no Sarcoma de Ewing. ${ }^{38-42}$ A literatura mostra que os sarcomas de partes moles são tumores com alto poder metastático e com grande potencial de recidiva local se não forem ressecados corretamente. ${ }^{16,17}$ Ao diagnóstico, aproximadamente $10 \%$ dos pacientes apresentam metástase e cerca de $50 \%$ dos pacientes com SPM irão desenvolvê-las. Menos de 20\% desses pacientes estarão vivos em 2 anos. Portanto, como a maioria dos SPM ocorrem nos membros, os ortopedistas serão provavelmente os primeiros profissionais a abordar essas lesões.

\section{Considerações Finais}

Em conclusão, é importante que qualquer tumor de partes moles seja submetido a avaliação de imagem adequada e, caso seja sólido e não adiposo, precisa ser submetido a biópsia com técnica adequada antes que seja realizada uma ressecção sem planejamento que possa comprometer o tratamento e o prognóstico do paciente.

Conflito de Interesses

Os autores declaram não haver conflito de interesses.

\section{Referências}

1 Fletcher CDM, Gronchi A. Tumors of soft tissue: Introduction. In: Fletcher CDM, Bridge JA, Hogendoorn PCW, Mertens F, editors. WHO Classification of Tumors of Soft Tissue and Bone. Fourth Edition. Geneva: The International Agency for Research on Cancer (IARC); 2013:14-18

2 Groisberg R, RoszikJ, Conley A, Patel SR, Subbiah V. The Role of NextGeneration Sequencing in Sarcomas: Evolution From Light Microscope to Molecular Microscope. Curr Oncol Rep 2017;19(12):78

3 Kirane A, Crago AM. The importance of surgical margins in retroperitoneal sarcoma. J Surg Oncol 2016;113(03):270-276

4 Siegel RL, Miller KD, Jemal A. Cancer statistics, 2019. CA Cancer J Clin 2019;69(01):7-34

5 Clark MA, Fisher C, Judson I, Thomas JM. Soft-tissue sarcomas in adults. N Engl J Med 2005;353(07):701-711

6 Brown HK, Schiavone K, Gouin F, Heymann MF, Heymann D. Biology of Bone Sarcomas and New Therapeutic Developments. Calcif Tissue Int 2018;102(02):174-195

7 Wibmer C, Leithner A, Zielonke N, Sperl M, Windhager R. Increasing incidence rates of soft tissue sarcomas? A population-based epidemiologic study and literature review. Ann Oncol 2010;21 (05):1106-1111

8 Trovik C, Bauer HCF, Styring E, et al. The Scandinavian Sarcoma Group Central Register: 6,000 patients after 25 years of monitoring of referral and treatment of extremity and trunk wall softtissue sarcoma. Acta Orthop 2017;88(03):341-347

9 Ogura K, Higashi T, Kawai A. Statistics of bone sarcoma in Japan: Report from the Bone and Soft Tissue Tumor Registry in Japan. J Orthop Sci 2017;22(01):133-143

10 Ogura K, Higashi T, Kawai A. Statistics of soft-tissue sarcoma in Japan: Report from the Bone and Soft Tissue Tumor Registry in Japan. J Orthop Sci 2017;22(04):755-764

11 Leal AL, Etchebehere M, Santos AO, et al. Evaluation of soft-tissue lesions with (18)F-FDG PET/CT: initial results of a prospective trial. Nucl Med Commun 2014;35(03):252-259

12 Widhe $\mathrm{B}$, Widhe $\mathrm{T}$. Initial symptoms and clinical features in osteosarcoma and Ewing sarcoma. J Bone Joint Surg Am 2000; 82(05):667-674

13 Ferrari A, Dirksen U, Bielack S. Sarcomas of Soft Tissue and Bone. Prog Tumor Res 2016;43:128-141

14 Kubo T, Furuta T, Johan MP, Ochi M. Prognostic significance of (18) F-FDG PET at diagnosis in patients with soft tissue sarcoma and bone sarcoma; systematic review and meta-analysis. Eur J Cancer 2016;58:104-111

15 Carbonnaux M, Brahmi M, Schiffler C, et al. Very long-term survivors among patients with metastatic soft tissue sarcoma. Cancer Med 2019;8(04):1368-1378 
16 Harati K, Goertz O, Pieper A, et al. Soft Tissue Sarcomas of the Extremities: Surgical Margins Can Be Close as Long as the Resected Tumor Has No Ink on It. Oncologist 2017;22(11):1400-1410

17 Hasley I, Gao Y, Blevins AE, Miller BJ. The Significance of a "Close” Margin in Extremity Sarcoma: A Systematic Review. Iowa OrthopJ 2018;38:123-130

18 Egas-Bejar D, Huh WW. Rhabdomyosarcoma in adolescent and young adult patients: current perspectives. Adolesc Health Med Ther 2014;5:115-125

19 Thway K. Sarcomas. In: Low KB. Brenner's Encyclopedia of Genetic. 2nd ed. Philadephia: Elsevier; 2013:327-331

20 Park JH, Kang CH, Kim CH, Chae IJ, Park JH. Highly malignant soft tissue sarcoma of the extremity with a delayed diagnosis. World J Surg Oncol 2010;8:84

21 Brouns F, Stas M, De Wever I. Delay in diagnosis of soft tissue sarcomas. Eur J Surg Oncol 2003;29(05):440-445

22 Cates JMM. The AJCC $8^{\text {th }}$ edition staging system for soft tissue sarcoma of the extremities or trunk: A Cohort study of the SEER database. J Natl Compr Canc Netw 2018;16(02):144-152

23 Nandra R, Forsberg J, Grimer R. If your lump is bigger than a golf ball and growing, think Sarcoma. Eur J Surg Oncol 2015;41(10): 1400-1405

24 Grimer RJ, Briggs TWR. Earlier diagnosis of bone and soft-tissue tumours. J Bone Joint Surg Br 2010;92(11):1489-1492

25 Taieb S, Ceugnart L, Gauthier H, Penel N, Vanseymortier L. [Soft tissue sarcoma: role of imaging for initial diagnosis and treatment]. Cancer Radiother 2006;10(1-2):22-33

26 Etchebehere EC, Hobbs BP, Milton DR, et al. Assessing the role of ${ }^{18} \mathrm{~F}$-FDG PET and ${ }^{18} \mathrm{~F}$-FDG PET/CT in the diagnosis of soft tissue musculoskeletal malignancies: a systematic review and metaanalysis. Eur J Nucl Med Mol Imaging 2016;43(05):860-870

27 Dancsok AR, Asleh-Aburaya K, Nielsen TO. Advances in sarcoma diagnostics and treatment. Oncotarget 2017;8(04):7068-7093

28 Hanasilo CE, Casadei MS, Auletta L, Amstalden EM, Matte SR, Etchebehere M. Comparative study of planned and unplanned excisions for the treatment of soft tissue sarcoma of the extremities. Clinics (São Paulo) 2014;69(09):579-584

29 Jernigan EW, Esther RJ. Soft tissue masses for the general orthopedic surgeon. Orthop Clin North Am 2015;46(03):417-428, xixi
30 George A, Grimer R. Early symptoms of bone and soft tissue sarcomas: could they be diagnosed earlier? Ann R Coll Surg Engl 2012;94(04):261-266

31 Pisters PWT, Leung DHY, Woodruff J, Shi W, Brennan MF. Analysis of prognostic factors in 1,041 patients with localized soft tissue sarcomas of the extremities. J Clin Oncol 1996;14(05):1679-1689

32 Baptista AM, Camargo OP, Croci AT, et al. Synovial sarcoma of the extremities: prognostic factors for 20 nonmetastatic cases and a new histologic grading system with prognostic significance. Clinics (São Paulo) 2006;61(05):381-386

33 Zumárraga JP, Batista FAR, Baptista AM, Caiero MT, Martino LPR de Camargo OP. Prognostic factors in patients with appendicular myxofibrosarcoma. Acta Ortop Bras 2018;26(05):320-324

$34 \mathrm{Wu}$ J, Qian S, Jin L. Prognostic factors of patients with extremity myxoid liposarcomas after surgery. J Orthop Surg Res 2019;14 (01):90

35 Tsuda Y, Ogura K, Kobayashi E, et al. Impact of geriatric factors on surgical and prognostic outcomes in elderly patients with softtissue sarcoma. Jpn J Clin Oncol 2017;47(05):422-429

36 Nakamura T, Katagiri H, Shido Y, et al. Analysis of Factors for Predicting Survival in Soft-tissue Sarcoma with Metastatic Disease at Initial Presentation. Anticancer Res 2017;37(06):3137-3141

37 Ishiraha HY, Jesus-Garcia Filho R, Korukian M, Ponte FM. Sarcomas de tecidos moles: fatores prognósticos. Rev Bras Ortop. 2004;39(11/12)

38 Anderson ME. Update on Survival in Osteosarcoma. Orthop Clin North Am 2016;47(01):283-292

39 Duchman KR, Gao Y, Miller BJ. Prognostic factors for survival in patients with high-grade osteosarcoma using the Surveillance, Epidemiology, and End Results (SEER) Program database. Cancer Epidemiol 2015;39(04):593-599

40 Kager L, Tamamyan G, Bielack S. Novel insights and therapeutic interventions for pediatric osteosarcoma. Future Oncol 2017;13 (04):357-368

41 Meazza C, Scanagatta P. Metastatic osteosarcoma: a challenging multidisciplinary treatment. Expert Rev Anticancer Ther 2016;16 (05):543-556

42 Macedo CR, Petrilli AS. Tumores ósseos malignos na criança e no adolescente. Pediatr Mod 1999;35(08):600-608 